\title{
Acute Changes in P-Wave Morphology by Pulmonary Vein Isolation in Atrial Fibrillation Patients
}

\author{
Ali Gharaviri ${ }^{1}$, Matthias Zink ${ }^{2}$, Mark Potse ${ }^{3}$, Simone Pezzuto ${ }^{1}$, Stef Zeemering ${ }^{2}$, Rolf Krause ${ }^{1}$, \\ Angelo Auricchio ${ }^{4}$, Ulrich Schotten ${ }^{2}$ \\ ${ }^{1}$ Center for Computational Medicine in Cardiology, Institute of Computational Science, Università della \\ Svizzera italiana, Lugano, Switzerland \\ ${ }^{2}$ Department of Physiology, Maastricht University, Maastricht, The Netherlands \\ ${ }^{3}$ Carmen team, Inria Bordeaux Sud-Ouest, Talence, France \\ ${ }^{4}$ Fondazione Cardiocentro Ticino, Lugano, Switzerland
}

\begin{abstract}
Pulmonary vein $(P V)$ plays an important role in atrial fibrillation $(A F)$ initiation, progression, and stability. Successful PV isolation (PVI), either by radiofrequency catheter or Cryoballoon ablation, may terminate $A F$ and prevent its recurrence. Whereas, incomplete $P V$ isolation or reconnection of isolated PVS underlies mechanisms of $A F$ recurrence. Hence, defining parameters able to predict a successful PVI and detect reconnections can assist clinicians in treatment of AF patients. Here, we developed a highly detailed human atrial model to simulate PVI and its acute effect on the P-wave morphology. Afterwards, the simulation results were compared and validated by recorded ECGs from patients before and after PVI procedure. In both simulation data and clinical recordings, we observed morphological changes in $P$ wave after PVI. More importantly our simulation helped us to find electrode positions, in which the differences in $P$-wave morphology before and after PVI were more pronounced.
\end{abstract}

\section{Introduction}

Atrial fibrillation (AF) is the most common sustained cardiac arrhythmia with a very high prevalence and associated with severe complications such as stroke [1,2]. The progression of AF from short-lived "paroxysmal" episodes to longer-lasting forms is a hallmark feature of this chronic disease with important clinical implications. Indeed, the longer a patient has been in AF, the more difficult it becomes to restore normal sinus rhythm, and treatment options extremely limited.[3] Therefore, making efficient AF prevention and therapy is a major clinical challenge.

Although mechanisms underlying AF initiation and perpetuation is not well understood, atrial muscle sleeves surrounding the pulmonary veins (PVs) are known to be the most frequent source of focal activities initiating atrial fibrillation (AF).[4] As a consequence, pulmonary veins
(PVs) play a significant role in atrial fibrillation initiation, progression, and stability. Therefore, $\mathrm{PV}$ isolation (PVI) is the therapy choice for patients with paroxysmal AF stubborn to antiarrhythmic therapies and patients with persistent AF.[5]

Despite the acute success of PVI in AF termination, specifically in paroxysmal AF patients, AF recurrences are not rare. $[6,7]$ More than $40 \%$ of patients experience AF recurrence one year after their PVI procedure[8], and in nearly all cases, with AF recurrence, one or more of the $\mathrm{PVs}$ is found to have re-established electrical connection to the atria. As a consequence, these patients may require additional catheter ablation[5, 9-11]. This influences patient's quality of life and risk for stroke.

Even though the mechanism underlying PV reconnections is not well understood, it is assumed to be caused by remained gaps in the line of blocks during PVI procedure or failure to generate a complete transmural block line. Therefore, finding non-invasive approach capable of detecting complete PVI and predicting PV reconnections during follow up, is highly desirable in clinical routines.[12]

Due to the presence of discontinuity in the left atria and PV connections there might be conduction delay between left atria and PV $[13,14]$. Therefore, it is possible that the final portion of P-wave reflects electrical activation of the $\mathrm{PVs}$ [14]. As a consequence, a successful PVI might be associated with a reduction in P-wave duration. Whereas, unsuccessful PVI might cause little or no changes in the Pwave durations.

In figure 1 an example of recorded ECG of a patient in three consecutive time points (pre PVI, acute post PVI and 3 months after) is illustrated. As it is shown in this figure, there were acute morphological changes in the second half of the P-wave. These morphological changes were stable after three months, and in this period the patient had neither an $\mathrm{AF}$ recurrence nor any symptoms related to AF.

Therefore, we hypothesized that morphological changes in the final part of the P-wave could serve as a predictor for a successful PV isolation or presence of reconnections. To test this hypothesis, we developed a highly detailed human 
atrial model and simulated circumferential PV isolation line. Afterwards, we compared the P-wave in the control simulations (without ablation lines) and PVI simulations. the time integral of the unfiltered signal between the onset and the end of the $\mathrm{P}$ wave.
A)

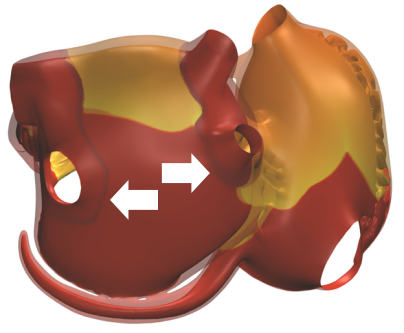

B)

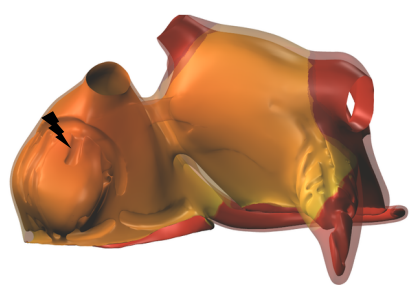

Figure 1. An example of Lead II ECG of a patient A) Pre-ablation. B) Acute post ablation (the area with a morphological change indicate by a red box). C) 3 months after ablation without $\mathrm{AF}$ recurrence after ablation

\section{Method}

\subsection{Human atria computer model}

We have developed a highly detailed human atrial model including epicardial layer and all major endocardial bundle structures. One to three layers of realistic fiber orientations were incorporated into the model, corresponding to their location in the atrium. The model consisted of approximately 5 million nodes spaced at $200 \mu \mathrm{m}$. Ionic currents for each node were described by the Courtemanche-Ramirez-Nattel human atrial cell model. [15] To simulate the ECG from the simulated atrial electrical activity, we embedded the atrial model in an inhomogeneous torso model. The torso model included lungs and interactivity blood masses. 256 body surface potentials were simulated using a bi-domain equation solved at 1-mm resolution.[16]

Two transmural ablation lines surrounding PVs positioned on the atrial model based on the documented method and localization of clinical ablation points (see figure 2). Virtual ablation lesions consisted of the tissue volumes within $0.35 \mathrm{~cm}$ (i.e., the ablation lesion radius for standard irrigated-tip catheters). [17] Along the ablation line intercellular coupling will be set to zero to avoid any conduction toward the PVs.[18, 19]. In both control (no ablation lines) and post ablation the normal propagation was initiated by injecting intracellular current in the region of the sino-atrial node.

\subsection{Analysis}

For all 256 electrograms, P-wave duration, P-wave amplitude and P-wave area were calculated. P-wave duration was defined as the interval between the stimulus time and the moment that all model elements became repolarized. P-wave amplitude was defined as the maximal vertical peak to peak distance. P-wave area was defined as
Figure 2. A) Position of ablation lines (white arrows). B) Anterior view of the human atrial model and the pacing location (black flash).

\section{Result}

In the simulated standard 12 lead ECG signals, there were slight morphological changes in the simulated Pwaves in PVI simulation compared with the P-waves in control simulation. These changes occurred at the second half of the P-wave. In figure 3, ECG leads with the largest differences between P-waves in control and PVI simulations is depicted.

For all 256 body surface electrograms in control and PVI simulations, P-wave area and amplitude were calculated. Averaged P-wave area for all 256 leads decreased from $1.3517 \pm 0.7193$ in control to $1.0832 \pm$ 0.5796 in PVI simulations. The averaged P-wave amplitude decreased as well from $0.0309 \mathrm{mV} \pm 0.0114$ in control to $0.0284 \mathrm{mV} \pm 0.0104$ in PVI simulations.
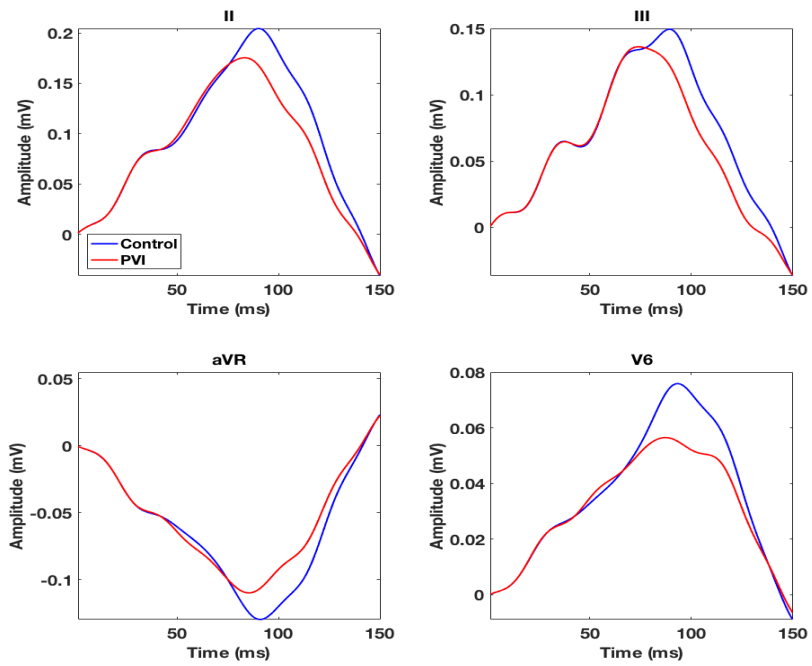

Figure 3. P-wave before (blue) and after (red) PVI in lead II, III, aVR, and V6. 
In order to quantify morphological differences between P-waves in control and PVI simulations, Euclidean distances between $\mathrm{P}$-waves pre and post ablations were calculated. In figure 4 projected color coded differences on the torso and simulated electrograms on the electrodes with largest differences illustrated. Highest differences were observed in the posterior side of the torso.
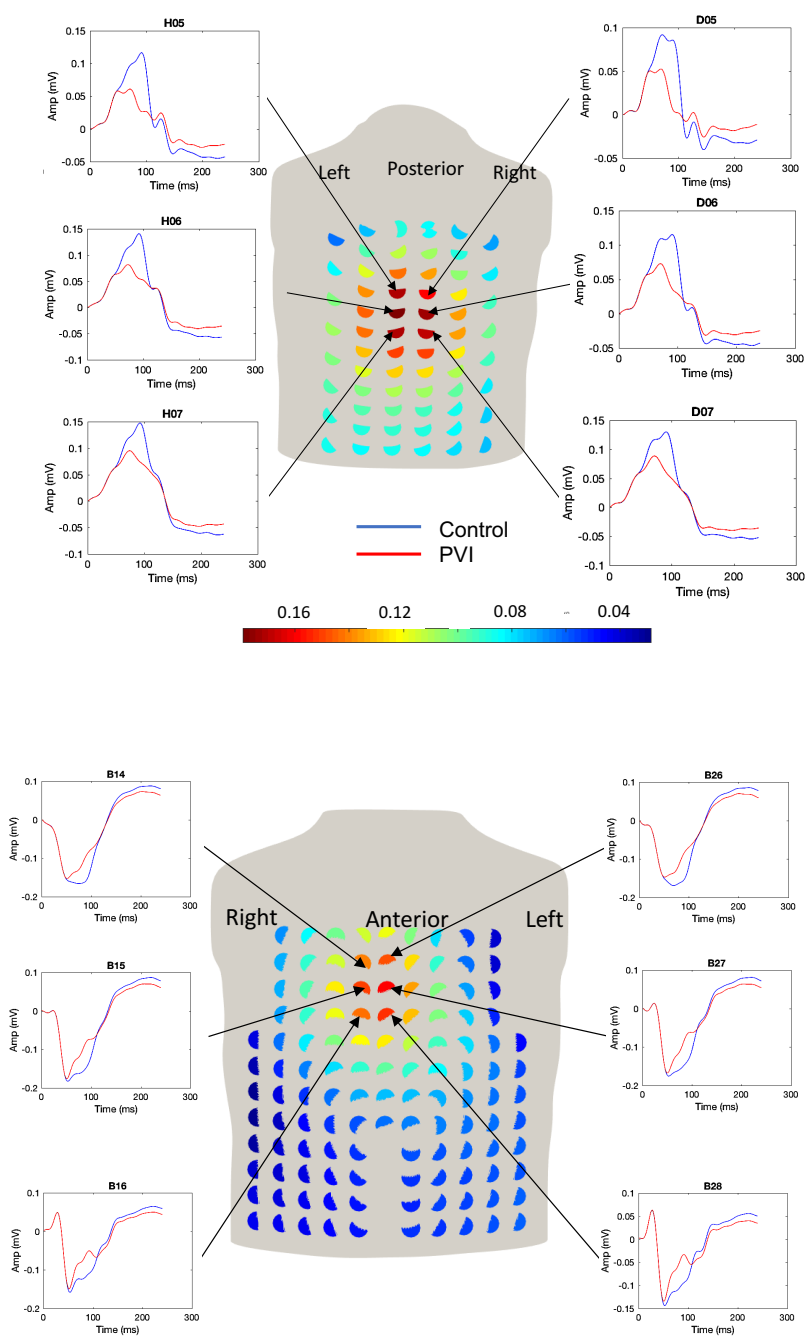

Figure 4. The Euclidean distance between pre- and postsimulated P-wave, color coded. Red shows the highest differences and blue shows the lowest difference. Top: Anterior view. Bottom: Posterior view.

\section{Discussion}

We have developed a highly detailed computer model of a human atria and torso to simulate PVI. The simulation results showed that a complete PVI led to a decrease in both the amplitude and the area of the P-wave. Notably, differences between $\mathrm{P}$-waves in control and PVI simulations were pronounced more in the posterior side of the torso rather than the anterior side. Recording electrograms from those locations are not considered in the clinical routines. Hence, analyzing those electrograms might be a great tool for clinicians to be sure that the PVI procedure is complete and to detect possible PV reconnections during the follow-up.

\section{Limitations}

Although the atrial model contains many details of atrial electrophysiology, the simulated activation sequence of our atrial model may not exactly reflect that of the patients in this study. The simulations were performed only on a generic computer model of the atria and torso, and anatomical variations were not included in this study. Thus, the simulated atrial electrogram morphology may not match the surface ECG of all patients. Finally, presence of fibrosis and slow conduction areas in the atria may cause changes in the atrial activation sequence. Therefore, PVs may not be the latest sites of activation.

\section{References:}

A. Loewe et al., "In-silico assessment of the dynamic effects of amiodarone and dronedarone on human atrial patho-electrophysiology," Europace, vol. 16 Suppl 4, pp. iv30-iv38, Nov 2014.

Y. Miyasaka et al., "Mortality trends in patients diagnosed with first atrial fibrillation: a 21-year community-based study," J Am Coll Cardiol, vol. 49, no. 9, pp. 986-92, Mar 62007.

$\begin{array}{llrl}\text { H. Calkins } & \text { et } & \text { al., } & \text { "2017 }\end{array}$ consensus statement on catheter and surgical ablation of atrial fibrillation: Executive summary," J Arrhythm, vol. 33, no. 5, pp. 369-409, Oct 2017. fibrillation by ectopic beats originating in the pulmonary veins," $N$ Engl J Med, vol. 339, no. 10, pp. 659-66, Sep 31998.

T. J. McGarry and S. M. Narayan, "The anatomical basis of pulmonary vein reconnection after ablation for atrial fibrillation: wounds that never felt a scar?," $\mathrm{J} \mathrm{Am}$ Coll Cardiol, vol. 59, no. 10, pp. 939-41, Mar 62012. A. Verma et al., "Approaches to catheter ablation for persistent atrial fibrillation," $N$ Engl J Med, vol. 372, no. 19, pp. 1812-22, May 72015.

L. Macle et al., "Adenosine-guided pulmonary vein isolation for the treatment of paroxysmal atrial fibrillation: an international, multicentre, randomised superiority trial," Lancet, vol. 386, no. 9994, pp. 6729, Aug 152015.

[8] H. Calkins et al., "2017 HRS/EHRA/ECAS/APHRS/SOLAECE expert consensus statement on catheter and surgical ablation of atrial fibrillation," Heart Rhythm, vol. 14, no. 10, pp. e275-e444, Oct 2017.

[9] D. J. Callans et al., "Efficacy of repeat pulmonary vein 
isolation procedures in patients with recurrent atrial fibrillation," J Cardiovasc Electrophysiol, vol. 15, no. 9, pp. 1050-5, Sep 2004.

[10] R. Cappato et al., "Prospective assessment of late conduction recurrence across radiofrequency lesions producing electrical disconnection at the pulmonary vein ostium in patients with atrial fibrillation," Circulation, vol. 108, no. 13, pp. 1599-604, Sep 30 2003.

[11] F. Ouyang et al., "Complete isolation of left atrium surrounding the pulmonary veins: new insights from the double-Lasso technique in paroxysmal atrial fibrillation," Circulation, vol. 110, no. 15, pp. 2090-6, Oct 122004.

[12] M. Saha et al., "Changes in P-wave morphology after pulmonary vein isolation: insights from computer simulations," Europace, vol. 18, no. suppl 4, pp. iv23iv34, Dec 2016.

[13] A. Hamabe et al., "Correlation between anatomy and electrical activation in canine pulmonary veins," (in English), Circulation, vol. 107, no. 11, pp. 1550-1555, Mar 252003.

[14] M. Ogawa et al., "Reduction of P-wave duration and successful pulmonary vein isolation in patients with atrial fibrillation," J Cardiovasc Electrophysiol, vol. 18, no. 9, pp. 931-8, Sep 2007.

[15] M. Courtemanche, R. J. Ramirez, and S. Nattel, "Ionic mechanisms underlying human atrial action potential properties: insights from a mathematical model," Am J Physiol, vol. 275, no. 1 Pt 2, pp. H301-21, Jul 1998.

[16] M. Potse et al., "Patient-specific modelling of cardiac electrophysiology in heart-failure patients," Europace, vol. 16 Suppl 4, pp. iv56-iv61, Nov 2014.

[17] S. M. Narayan, D. E. Krummen, K. Shivkumar, P. Clopton, W. J. Rappel, and J. M. Miller, "Treatment of atrial fibrillation by the ablation of localized sources: CONFIRM (Conventional Ablation for Atrial Fibrillation With or Without Focal Impulse and Rotor Modulation) trial," J Am Coll Cardiol, vol. 60, no. 7, pp. 628-36, Aug 142012.

[18] L. Dang et al., "Evaluation of ablation patterns using a biophysical model of atrial fibrillation," Ann Biomed Eng, vol. 33, no. 4, pp. 465-74, 2005.

[19] M. Reumann, J. Bohnert, G. Seemann, B. Osswald, and O. Dossel, "Preventive ablation strategies in a biophysical model of atrial fibrillation based on realistic anatomical data," IEEE Trans Biomed Eng, vol. 55, no. 2, pp. 399-406, 2008.

Address for correspondence:

Ali Gharaviri, Center for Computational Medicine in Cardiology, Institute of Computational Science, Università della Svizzera italiana.

Via Buffi 13, 6900 Lugano, Switzerland.

ali.gharaviri@usi.ch. 